\title{
PbrSLAH3 is a nitrate-selective anion channel which is modulated by calcium- dependent protein kinase 32 in pear
}

\author{
Guodong Chen ${ }^{\dagger}$, Li Wang ${ }^{\dagger}$, Qian Chen, Kaijie Qi, Hao Yin, Peng Cao, Chao Tang, Xiao Wu, Shaoling Zhang, \\ Peng Wang ${ }^{*}$ and Juyou Wu ${ }^{*}$ (i)
}

\begin{abstract}
Background: The functional characteristics of SLAC/SLAH family members isolated from Arabidopsis thaliana, poplar, barley and rice have been comprehensively investigated. However, there are no reports regarding SLAC/SLAH family genes from Rosaceae plants.

Results: In this study, the function of PbrSLAH3, which is predominately expressed in pear (Pyrus bretschneideri) root, was investigated. PbrSLAH3 can rescue the ammonium toxicity phenomenon of slah3 mutant plants under high-ammonium/low-nitrate conditions. In addition, yeast two-hybrid and bimolecular fluorescence complementation assays confirmed that PbrSLAH3 interacts with PbrCPK32. Moreover, when PbrSLAH3 was co-expressed with either the Arabidopsis calcium-dependent protein kinase (CPK) 21 or PbrCPK32 in Xenopus oocytes, yellow fluorescence was emitted from the oocytes and typical anion currents were recorded in the presence of extracellular $\mathrm{NO}_{3}{ }^{-}$. However, when PbrSLAH3 alone was injected, no yellow fluorescence or anion currents were recorded, suggesting that anion channel PbrSLAH3 activity was controlled through phosphorylation. Finally, electrophysiological and transgene results showed that PbrSLAH3 was more permeable to $\mathrm{NO}_{3}{ }^{-}$than $\mathrm{Cl}^{-}$.
\end{abstract}

Conclusion: We suggest that PbrSLAH3 crossing-talk with PbrCPK32 probably participate in transporting of nitrate nutrition in pear root.

Keywords: Nitrogen, Signaling, Anion channel, Nitrate, PbrSLAH3, Phosphorylation

\section{Background}

Anion channels, which widely exist in a variety of bio-membranes, play an important role in cell signaling, osmoregulation, metabolism, stress tolerance and plant nutrition [1-4]. Electrophysiological data from different research groups confirmed that rapid-type (R-type) and slow-type (S-type) anion channels are involved in anion transport [5-7]. By far, SLAC/SLAH family genes (S-type anion channels) from species, including Arabidopsis thaliana, poplar, barley and rice, have been identified and studied, five members in Arabidopsis [8, 9], seven members in Populus [10] and nine members in rice [11].

\footnotetext{
* Correspondence: wangpeng@njau.edu.cn; juyouwu@njau.edu.cn †Guodong Chen and Li Wang contributed equally to this work. Center of Pear Engineering Technology Research, State Key Laboratory of Crop Genetics and Germplasm Enhancement, College of Horticulture, Nanjing Agricultural University, No 6. Tongwei Road, Nanjing, China
}

However, no reports regarding $S L A C / S L A H$ family genes from Rosaceae plants have been found.

To date, only the physiological functions of the SLAC/ $S L A H$ family members in Arabidopsis have been widely studied [12-15]. AtSLAC1, together with SLAC1 homologous 3 (AtSLAH3), plays an important role in regulating ABA-induced stomatal closure [16-19]. During this process, $A B A$ is firstly perceived by the RCAR/PYR/ PYL-ABI1 complex, which then releases protein kinase from $A B I 1$-induced inhibition, and then AtSLAC1 and AtSLAH3 are activated by the calcium-independent protein kinase OST1 and the calcium-dependent protein kinases (CPKs) of Arabidopsis [13]. Meanwhile, other members of the SLAC/SLAH family in Arabidopsis have also been intensively studied. The results from the Geiger group showed that SLAH1, as a silent subunit, facilitates SLAH3-mediated chloride efflux from pericycle

(c) The Author(s). 2019 Open Access This article is distributed under the terms of the Creative Commons Attribution 4.0 International License (http://creativecommons.org/licenses/by/4.0/), which permits unrestricted use, distribution, and 
cells into the root xylem vessels [20,21]. When co-expression the silent SLAH1 subunit and SLAH3 in Xenopus oocytes, SLAH3 was activated even in the absence of nitrate and calcium-dependent protein kinases [20]. Besides, $S L A H 2$, another member of the SLAC/SLAH family, which is expressed in the stele of the root, acts in a nitrate-specific channel that is impermeable to chloride when co-expressed with protein kinases in Xenopus oocytes [22].

Although AtSLAC1 and AtSLAH3 exhibit similar physiological functions in guard cells, different expression patterns and functional characteristics were observed between these two channels. For instance, AtSLAC1 is exclusively expressed in the guard cells [23], while AtSLAH3, in addition to being expressed in the guard cells, is also predominantly expressed in the root and weakly expressed in the pollen tube [24, 25]. Furthermore, AtSLAC1 exhibits a high permeability to $\mathrm{NO}_{3}{ }^{-}$than $\mathrm{Cl}^{-}$, with a $\mathrm{P}\left(\mathrm{NO}_{3}{ }^{-}\right) / \mathrm{P}\left(\mathrm{Cl}^{-}\right)$ratio of 10 [22], while AtSLAH3 is a nitrate-activated S-type anion channel with a $\mathrm{P}\left(\mathrm{NO}_{3}{ }^{-}\right) / \mathrm{P}\left(\mathrm{Cl}^{-}\right)$ratio of $20[13,14]$. Apart from regulating the stomatal closure together with AtSLAC1, AtSLAH3 alleviates ammonium toxicity and regulates pollen tube growth in Arabidopsis [25].

More importantly, SLAC/SLAH family genes from different plant species with high similarity showed varied expression patterns and functional characteristics. For instance, in contrast to AtSLAC1, which is permeable to both chloride and nitrate, OsSLAC1 is identified as a nitrate-selective anion channel [11]. Furthermore, in contrast to AtSLAH3, which is predominantly expressed in the root and is dependent on phosphorylation activation, PttSLAH3, which is predominantly expressed in poplar secretory epithelia, is independent of activation by protein kinases [10]. Thus, based on these results mentioned above, we speculated that SLAH3 isolated from the pear, which has not been clarified until recently, may own special functional-characteristics.

Nitrate is the major source of nitrogen for plants in nature and in agriculture under aerobic conditions $[14,26,27] . S L A C / S L A H$ genes from different species have been proven to play an important role in nitrate transport. In this study, we focused on investigating the function of PbrSLAH3, which is predominantly expressed in pear root verified by RT-PCR, qRT-PCR and $\beta$-Glucuronidase (GUS) staining assays. The physiological function of PbrSLAH3 was investigated by its over-expression in a slah3 mutant. In consistent with that of AtSLAH3, PbrSLAH3 also participates in alleviation of ammonium toxicity under high ammonium/low nitrate conditions. Furthermore, both yeast two-hybrid and bimolecular fluorescence complementation (BiFC) assays confirmed the interaction between PbrSLAH3 and PbrCPK32. Finally, an electrophysiological experiment revealed that PbrSLAH3 is activated by PbrCPK32 and the channel is highly selective for nitrate without obvious permeability to chloride. Thus, we conclude that PbrSLAH3 serve as a nitrate efflux channel and may participate in nitrate transport in pear root.

\section{Results \\ The phylogeny and expression patterns of SLAC/SLAH genes in pear}

AtSLAC/SLAH orthologous in pear were identified by a BLASTp algorithm-based search of the pear genomic database (http://peargenome.njau.edu.cn/) using the amino acid sequences of AtSLAC/SLAH as queries. To compare the similarity levels between the PbrSLAC/ $S L A H$ and AtSLAC/SLAH, a rooted phylogenetic tree was constructed using the neighbor-joining method (Fig. 1a). As shown in Fig. 1, PbrSLAC1 and AtSLAC1 were grouped to the same branch, while other three PbrSLAC/SLAH proteins, together with AtSLAH2 and AtSLAH3, belonged to another branch. Orthologs of AtSLAC/SLAH in pear did not phylogenetically cluster with AtSLAH1 and AtSLAH4. To further determine which genes in the PbrSLAC/SLAH family may participate in nitrate transporting in pear root, the expression patterns of PbrSLAC/SLAH family genes in the root, leaf, pollen grain and pollen tube were analyzed by quantitative and semi-quantitative RT-PCR (Fig. $1 \mathrm{~b}$ and c). PbrSLAC1 was exclusively expressed in leaf, while PbrSLAH3, PbrSLAH2/3-2 and PbrSLAH2/3-3 were widely expressed in root, leaf and pollen. PbrSLAH3 displayed a relative higher expression level than the other $\mathrm{PbrSLAH} 2 / 3$ genes in pear root (Fig. 1b). Furthermore, the transcriptome data analysis suggested that the expression level of PbrSLAH3 was up-regulated during nitrate starvation and then, after the re-supply of nitrate, its expression level was gradually down-regulated (Additional file 1: Figure S1).Taken together these results, we presume that PbrSLAH3 may be involved in nitrate transport in pear root and may play an important role in nitrate nutrition in pear trees.

\section{Subcellular localization and accurate organizational positions of PbrSLAH3}

To determine the subcellular localization of PbrSLAH3, the fusion gene of PbrSLAH3-GFP was constructed and transformed into tobacco (N. benthamiana) leaves. As previously reported, the control GFP was uniformly distributed throughout the whole cell, and the PbrSLAH3GFP fusion protein was observed exclusively in the plasma membrane with a Zeiss LSM 780 Image Browser (Fig. 2a). To further identify the accurate expression profiles of PbrSLAH3 in pear, transgenic Arabidopsis plants carrying a GUS gene under control of the PbrSLAH3 promoter fragment (2000 bp) were generated. The GUS activity assays 


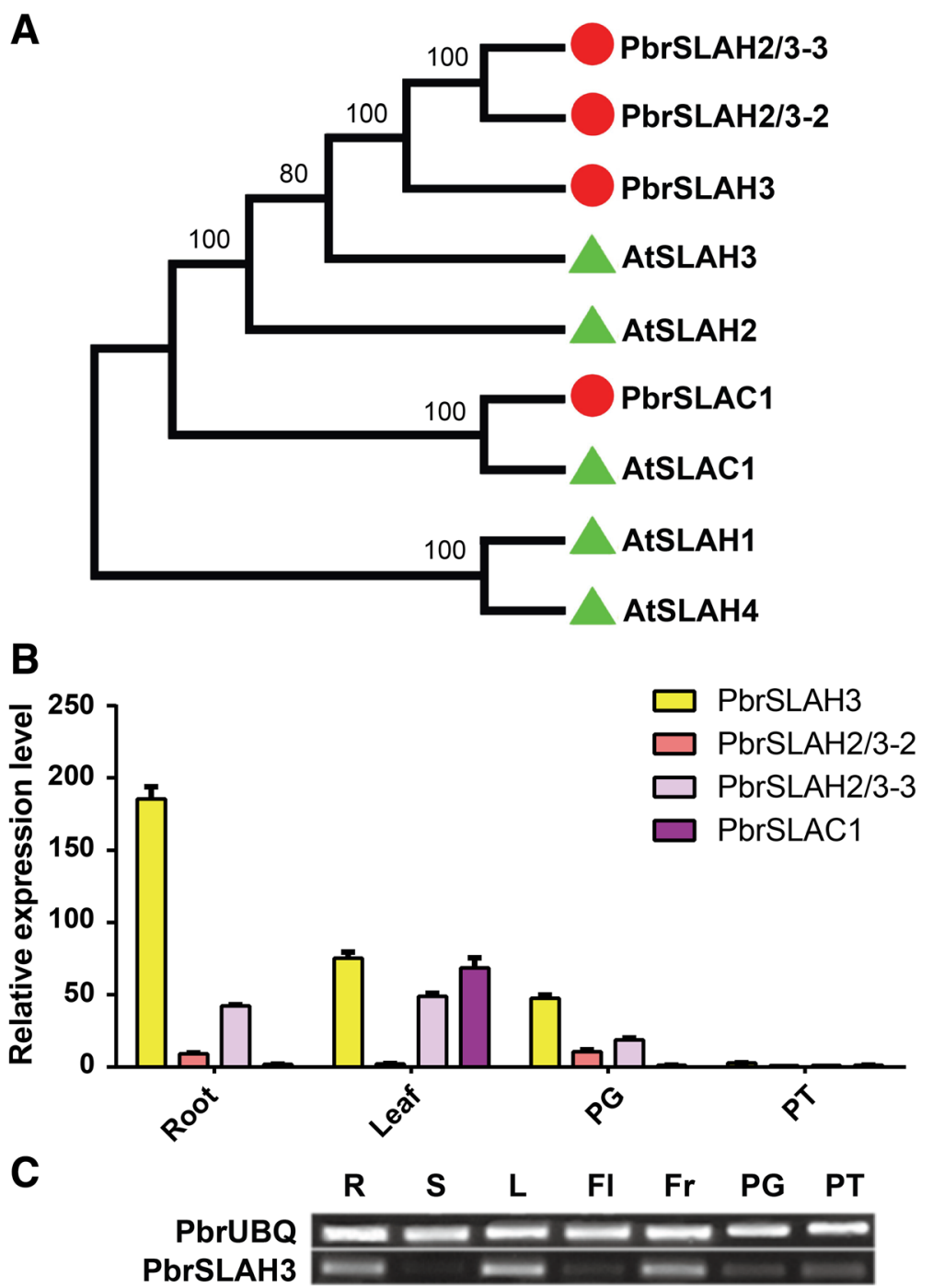

Fig. 1 The determination of S-type anion channels in pear and the expression analysis. a Phylogenetic tree of the Arabidopsis thaliana SLAC/SLAH anion channel family and the pear orthologs. Four putative PbrSLAC/SLAH anion channels were identified in the Pyrus genome database. PbrSLAC1 appears to be related to AtSLAC1, other three homologs were classified into the AtSLAH2/3 group, while AtSLAH1/4 homologs could not be identified in pear. The PbrSLAH2/3-1 sequence was used to clone the ortholog from Pyrus bretschneideri and named PbrSLAH3. b The expression patterns of PbrSLAC/SLAH family genes in the root, leaf, pollen grain and pollen tube of pear were analyzed by qRT-PCR. The values displayed in the figure were the geometric means of the relative expression of PbrSLAC/SLAH when both PbrTUB and PbrUBQ as reference genes. Three biological and three technical replicates were processed for the GRT-PCR assays. The error bars indicate standard deviations. The data are shown as mean values \pm SDs. c RT-PCR was used to confirm the expression patterns of the PbrSLAH3 gene and PbrUBQ gene was used as an internal control

showed that PbrSLAH3 is strongly expressed in roots, young leaves, mature leaves and stoma, and is weakly expressed in stems. However, it is not expressed in seed and silique (Fig. 2b-g). Thus, we presume that PbrSLAH3 may have diverse functions similar to those of AtSLAH3, which plays an important role in the alleviation of ammonium toxicity and regulation of stomatal closure and pollen growth.

\section{Functional characterization of PbrSLAH3 in Arabidopsis}

In line with previous reports, the Arabidopsis slah3-3 mutant exhibited more severe ammonium toxicity than wild-type when grown on plates containing a high-ammonium/low-nitrate medium [25]. As shown in Fig. 3a, the T-DNA insertion site in the slah3-3 mutant was in the second intron of AtSLAH3. To examine the physiological function of PbrSLAH3, complementation lines of the Arabidopsis slah3-3 mutant were constructed by the gene of PbrSLAH3. Two transgenic lines were selected based on the RT-PCR results. The mRNA of PbrSLAH3 was not detected in wild-type and slah3-3 mutant plants, but it was highly expressed in the transgenic lines (Fig. 3b). Phenotype testing showed that the ammonium 


\section{A}
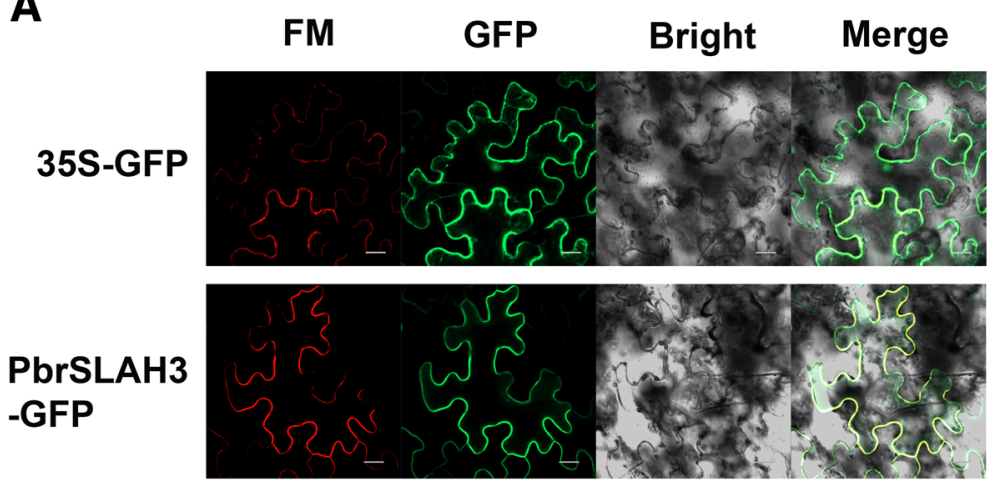

B

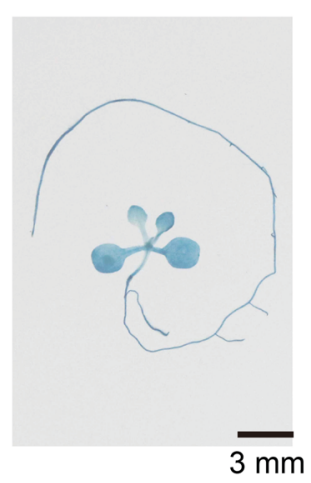

C

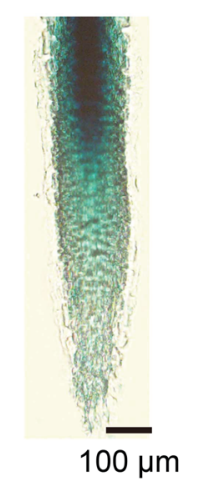

D

E

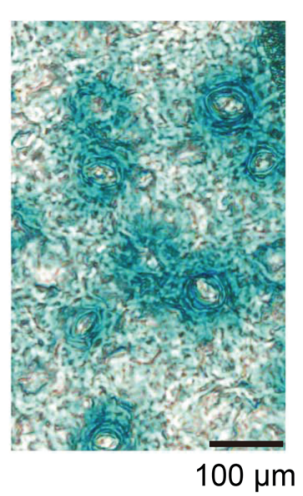

F

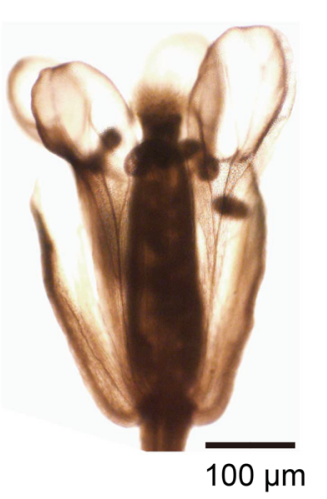

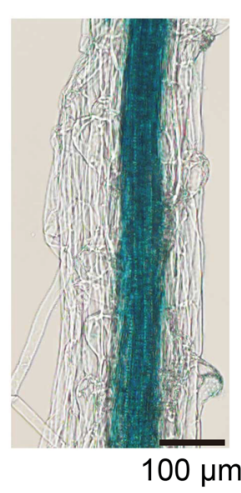

G

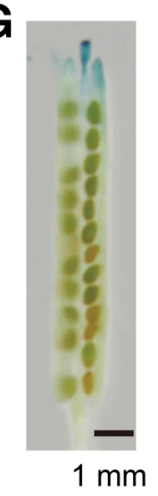

Fig. 2 The subcellular localization of PbrSLAH3 to the plasma membrane, and a histochemical analysis of GUS activity in PbrSLAH3 transgenic Arabidopsis (Col-0) plants. a GFP fluorescence emitted from the leaves of tobacco infected by Agrobacterium harboring PbrSLAH3-GFP was detected under a confocal microscope and the plasma membranes are stained red by FM4-64. GFP: green fluorescent protein; Chl: chlorophyll; Bright: bright-field image of Agrobacterium tumefaciens-infiltrated tobacco leaves; Merge: merged fluorescent images; Bar $=10 \mu \mathrm{m}$. b-g GUShistochemical analysis performed with Arabidopsis plants expressing the reporter gene under the control of the PbrSLAH3 promoter. PbrSLAH3 was expressed in young leaves, mature leaves, roots, stems, epidermal hairs, stomatal and siliques

toxicity phenotype of the slah3-3 mutant under high-ammonium/low-nitrate conditions was rescued by the overexpression of PbrSLAH3 (Fig. 3c and Additional file 1: Figure S2). Statistical results demonstrated that the root length of the slah3-3 mutant was approximately $56-70 \%$ that of the wild-type when grown on high $\mathrm{NH}_{4}^{+}$medium supplied with 0 or $0.1 \mathrm{mM} \mathrm{KNO}_{3}$, while there were no differences between each of the complementation lines and the wild-type (Fig. 3d-g), suggesting that PbrSLAH3 plays an important role in the alleviation of ammonium toxicity under nitrate-limited conditions. Furthermore, when the $\mathrm{KNO}_{3}$ concentration in the medium reaching up to $1 \mathrm{mM}$ or more $(20 \mathrm{mM})$, no clear differences were observed between the slah3-3 mutant and wild-type, indicating that sufficient levels of nitrate can counteract ammonium toxicity. 
A
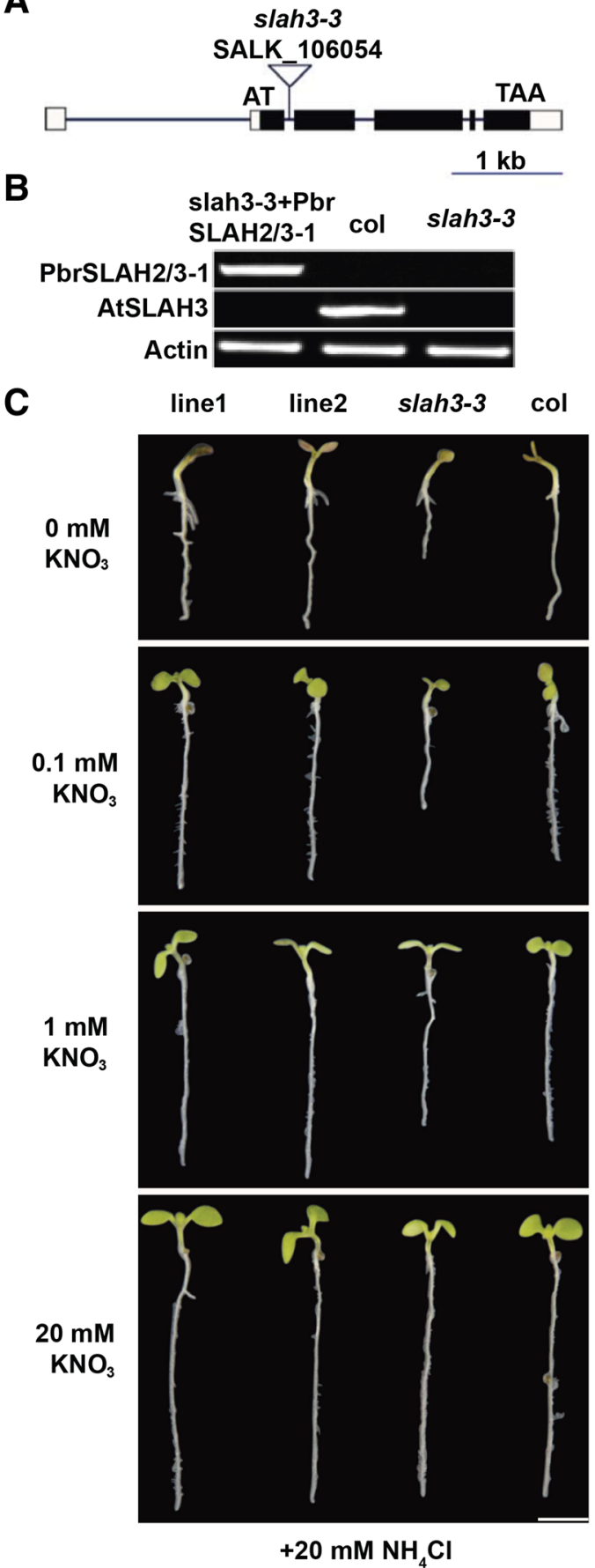

D
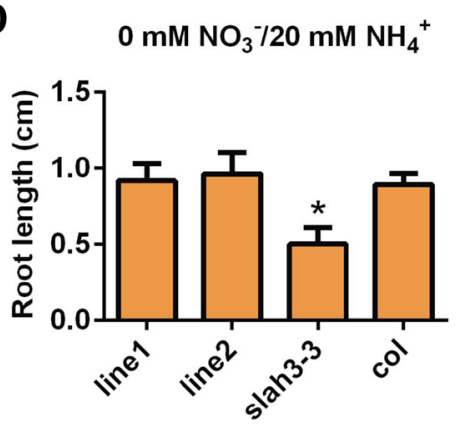

E

$0.1 \mathrm{mM} \mathrm{NO}_{3}{ }^{-} / 20 \mathrm{mM} \mathrm{NH}_{4}{ }^{+}$

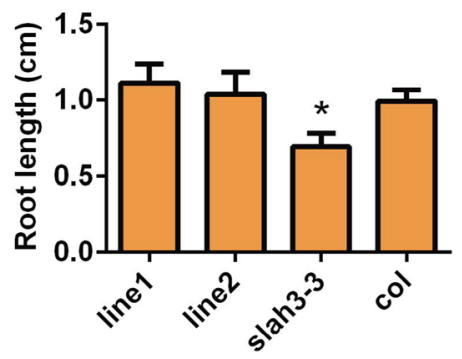

$\mathbf{F}$ $1 \mathrm{mM} \mathrm{NO}_{3}{ }^{-} / 20 \mathrm{mM} \mathrm{NH}_{4}^{+}$

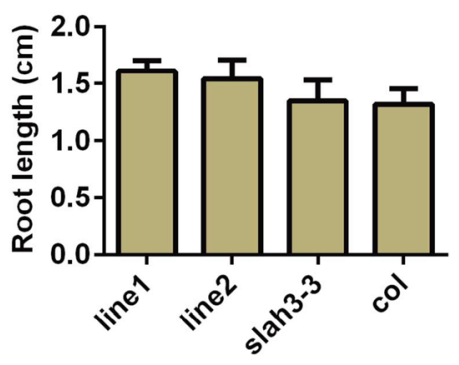

G $\quad 20 \mathrm{mM} \mathrm{NO}_{3}{ }^{-} / 20 \mathrm{mM} \mathrm{NH}_{4}^{+}$

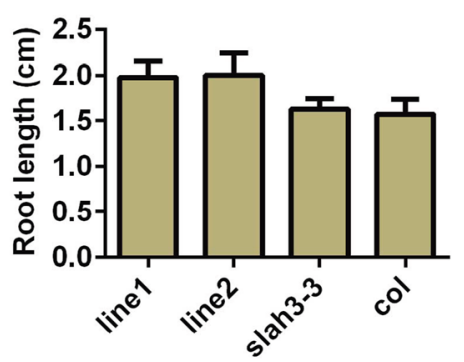

Fig. 3 PbrSLAH3 rescues the ammonium toxicity of slah3-3 mutant plants under high ammonium/low-nitrate conditions. a Schematic map of the T-DNA insertion site in the slah3-3 mutant. b RT-PCR analyses of PbrSLAH3 and AtSLAH3 expression levels in transgenic, wild-type (Col-0) and slah3-3 mutant plants. Actin was used as a control. c Growth comparison among 7-d-old transgenic lines 1 and 2, wild-type (Col-0) and slah3-3 mutant seedlings grown on 1/2 N-free Murashige and Skoog medium supplemented with different concentrations of $\mathrm{KNO}_{3}(0-20 \mathrm{mM})$ and 20 $\mathrm{mM} \mathrm{NH}_{4} \mathrm{Cl}$. $\mathbf{d}-\mathbf{g}$ The average primary root lengths of seedlings under the same conditions as displayed in (c) were statistical analyzed. Results are averages \pm SEs of three independent experiments ( $n=15$ per experiment). The asterisk represents statistical significance among transgenic lines, wild-type and slah3-3 mutant plant (Student's t-test, $\left.{ }^{*} P<0.05\right)$. Bar $=0.5 \mathrm{~cm}$ 


\section{Identification of PbrSLAH3 regulators}

Considering the activation of the AtSLAH3-encoded channel requires the protein kinase AtCPK21 [10, 13], and $\mathrm{PbrSLAH} 3$ shows high similarity and similar function to that of AtSLAH3, we investigated whether PbrSLAH3 can interact with AtCPK21 to transport nitrate ions. BiFC assays showed that PbrSLAH3 can interact with AtCPK21 in the plasma membrane of tobacco epidermal cells (Additional file 1: Figure S3), indicating that PbrSLAH3 may be involved in a signal-transduction pathway through phosphorylation and it may be a counterpart of AtSLAH3.

Given PbrSLAH3 is actived by AtCPK21, we hypothesized that PbrSLAH3 may be regulated by orthologes of AtCPK21 in pear. We initially constructed a phylogenetic tree based on DNA sequences in pear to analyze associations between PbrSLAH3 and CPKs owing to the characteristic interactions between CPKs and anion channel proteins in Arabidopsis (Additional file 1: Figure S4 and Table S2). We identified 17 candidate PbrCPK proteins that may interact with PbrSLAH3 in pear. Then, a yeast two-hybrid experiment was firstly performed to assess the ability of PbrSLAH3 to interact with each of the PbrCPKs. The full-length of PbrSLAH3 fused with the pDHB-C and the PbrCPKs fused with the pPR3-N vector were then co-transformed into the yeast strain NMY51 using the lithium acetate method, respectively. Co-transformed yeast cells were first selected on plates containing medium lacking Trp and Leu to confirm the introduction of the PbrSLAH3-pDHB-C and PbrCPKspPR3-N plasmids into the yeast cells (Additional file 1: Figure S5). The positive clones were then transferred to selective plates lacking Trp, Leu, His and Ade. As shown in Additional file 1: Figure S5, yeast cells co-transformed with the positive controls (NubG and $\mathrm{pDHB}-\mathrm{C}$ ) and interactive genes (PbrSLAH3-pDHB-C and PbrCPK32-pPR3-N) could grow on the selective medium (SD/-Trp-Leu-His-Ade). However, yeast cells harboring PbrSLAH3-pDHB-C and the other PbrCPKs-pPR3-N plasmids could not grow on the selective media. To further confirm the interaction between PbrSLAH3 and PbrCPK32, control experiments were performed to rule out self-activation. As shown in Fig. 4a, neither yeast cells harboring PbrSLAH3-pDHB-C and pPR3-N nor pDHB-C and $P b r C P K 32-\mathrm{pPR} 3-\mathrm{N}$ could grow on the selective media. Only PbrSLAH3-pDHB-C and PbrCPK32-pPR3-N together could grow on the selective media (SD/-Trp-Leu-His-Ade; Fig. 4a). In addition, PbrCPK32 is mainly expressed in the root system, and the subcellular localization study displayed that the PbrCPK32-GFP fusion protein is mostly distributed in the cell membrane (Additional file 1: Figure S6). In all, these results revealed that PbrSLAH3 could interact with PbrCPK32 in the plasma membrane.
To further confirm the interaction between PbrSLAH3 and PbrCPK32 in vivo, BiFC assays was performed (Fig. 4b). PbrSLAH3 tagged with a split YFP $^{\mathrm{N}}$-terminal fragment (nYFP) and PbrCPK32 tagged with an $\mathrm{YFP}^{\mathrm{C}}$-terminal fragment (cYFP) were transiently co-infiltrated into epidermal cells of $N$. benthamiana leaves using Agrobacterium. As indicated in Fig. 4b, a strong YFP fluorescent signal was detected in the plasma membrane of epidermal cells when co-expression

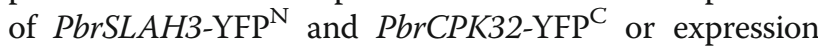
35S-YFP alone (as a positive control), while no YFP fluorescent signal was observed in cells co-expressing of PbrSLAH3-YFP ${ }^{\mathrm{N}}$ and $\mathrm{YFP}^{\mathrm{C}}$ or $\mathrm{PbrCPK} 32-\mathrm{YFP}^{\mathrm{C}}$ and $\mathrm{YFP}^{\mathrm{N}}$ (Additional file 1: Figure S7). Thus, the BiFC assays further confirmed the interaction between PbrSLAH3 and PbrCPK32 in the plasma membrane.

\section{PbrSLAH3 mediates S-type anion currents when co- expressed with CPKs in Xenopus oocytes}

Since AtCPK21 interacts with PbrSLAH3, we further tested whether this protein kinase regulates the activity of PbrSLAH3. When this putative anion channel was expressed alone in Xenopus oocytes, no YFP fluorescence signal was detected and no anion currents could be measured (Fig. 5a and Additional file 1: Figure S8). However, when PbrSLAH3 was co-expressed with AtCPK21, YFP fluorescence was emitted from oocytes and typical anion currents were recorded in the presence of $\mathrm{NO}_{3}^{-}$with membrane polarization (Fig. 5a).

In line with the functional characteristics of AtSLAH3, which is a preferential $\mathrm{NO}_{3}{ }^{-}$-permeable channel that is 20 times more permeable to $\mathrm{NO}_{3}{ }^{-}$than $\mathrm{Cl}^{-}, \mathrm{PbrSLAH} 3$ is also a preferential $\mathrm{NO}_{3}{ }^{-}$-permeable channel. The magnitudes of the anion currents were greater in the nitrate-bath medium than that under the same concentration of chlorine. In addition, the anion currents were largely dependent on the extracellular $\mathrm{NO}_{3}{ }^{-}$concentrations (Fig. 5b). As shown in Fig. 5c, typical anion currents were elicited from oocytes in the presence of 25 $\mathrm{mM}$ nitrate medium. Although they were rather low, the anion currents increased 133 and $266 \%$ when the $\mathrm{NO}_{3}{ }^{-}$ concentration reached up to $50 \mathrm{mM}$ and $100 \mathrm{mM}$, respectively. Together, in the presence of extracellular nitrate solution, PbrSLAH3 mediates S-type anion currents when co-expressed with AtCPK21 in Xenopus oocytes.

Due to PbrSLAH3 was activated by AtCPK21 and an interaction was observed between PbrSLAH3 and PbrCPK32, we hypothesized that the activity of PbrSLAH3 was regulated by PbrCPKs in pear. To test this hypothesis, PbrSLAH3 was injected alone or coinjected with PbrCPK32 in the Xenopus oocytes. PbrSLAH3 injected alone could not mediate the anion currents and no specific YFP fluorescence was emitted from Xenopus oocytes 


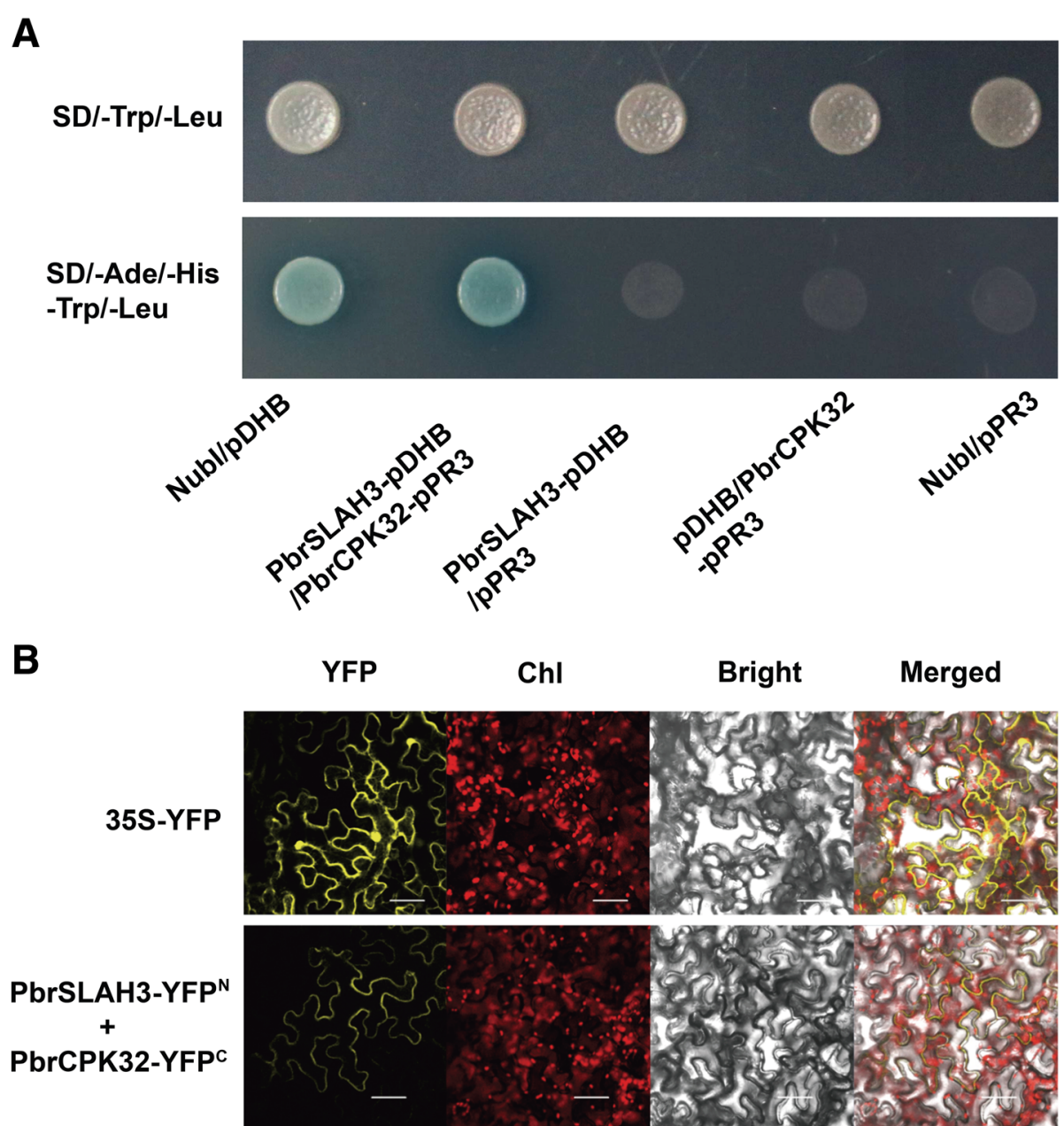

Fig. 4 Interaction between PbrSLAH3 and PbrCPK32 as assessed by yeast two-hybrid and bimolecular fluorescence complementation assays. a Yeast strain NMY51 co-transformed with the PbrSLAH3 bait vector and PbrCPK32 prey vector, along with a positive control (co-transformed Nubl vector and pDHB vector) were grown in medium lacking Ade, His, Trp and Leu. The negative control could not grow in the same medium. $\mathbf{b}$ YFP indicates yellow fluorescence protein; Chl: chlorophyll; Bright: bright-field image of Nicotiana benthamiana leaves infiltrated with Agrobacterium tumefaciens; Merge: digital merge of bright field and fluorescent images. Bar $=10 \mu \mathrm{m}$

(Fig. 6a and Additional file 1: Figure S8). However, a YFP fluorescence signal was detected with a laser microscope (Fig. 6a) and macroscopic currents were elicited in the Xenopus oocytes when co-expression the PbrSLAH3 with PbrCPK32 (Fig. 6b). The I-V curves plotted against different concentrations of $\mathrm{NO}_{3}{ }^{-}$showed that the magnitudes of the anion currents mediated by PbrSLAH3 were strongly dependent on the external $\mathrm{NO}_{3}{ }^{-}$concentrations. However, only small macroscopic currents were induced by extracellular $\mathrm{Cl}^{-}$. Furthermore, the rate of $\mathrm{NO}_{3}{ }^{-}$decrements was higher for 35S:PbrSLAH3 plants than that of the slah3-3 mutant Arabidopsis, based on $\mathrm{NO}_{3}{ }^{-}$measurements from culture solution (Additional file 1: Figure S9). However, the rate of $\mathrm{Cl}^{-}$decrements was barely changed between 35S:PbrSLAH3 plants and slah3-3 mutant Arabidopsis, based on $\mathrm{Cl}^{-}$measurements from culture solution (Additional file 1: Figure S9), which indicated that overexpression of $\mathrm{PbrSLAH} 3$ was greatly permeable $\mathrm{NO}_{3}{ }^{-}$ than $\mathrm{Cl}^{-}$in the root. Taken together these results demonstrated that PbrSLAH3 was activated by PbrCPK32 and exhibited highly nitrate selectivity without obvious permeability to chloridion.

\section{Discussion}

Nitrate is an important nutrient, and is also widely used as a signaling molecule during plant growth and development [14]. Following the up-take of nitrate by plasma membrane-localized nitrate transporters, nitrate is translocated into the vasculature for long-distance transport. It has been reported that $S L A C / S L A H$ family genes could meditate the $\mathrm{NO}_{3}{ }^{-}$efflux and may participate in $\mathrm{NO}_{3}{ }^{-}$transport $[20,22,25]$. Considering AtSLAC1 is exclusively expressed in guard cells, the other four AtSLAH (SLAH1 to SLAH4) genes, which are predominantly 


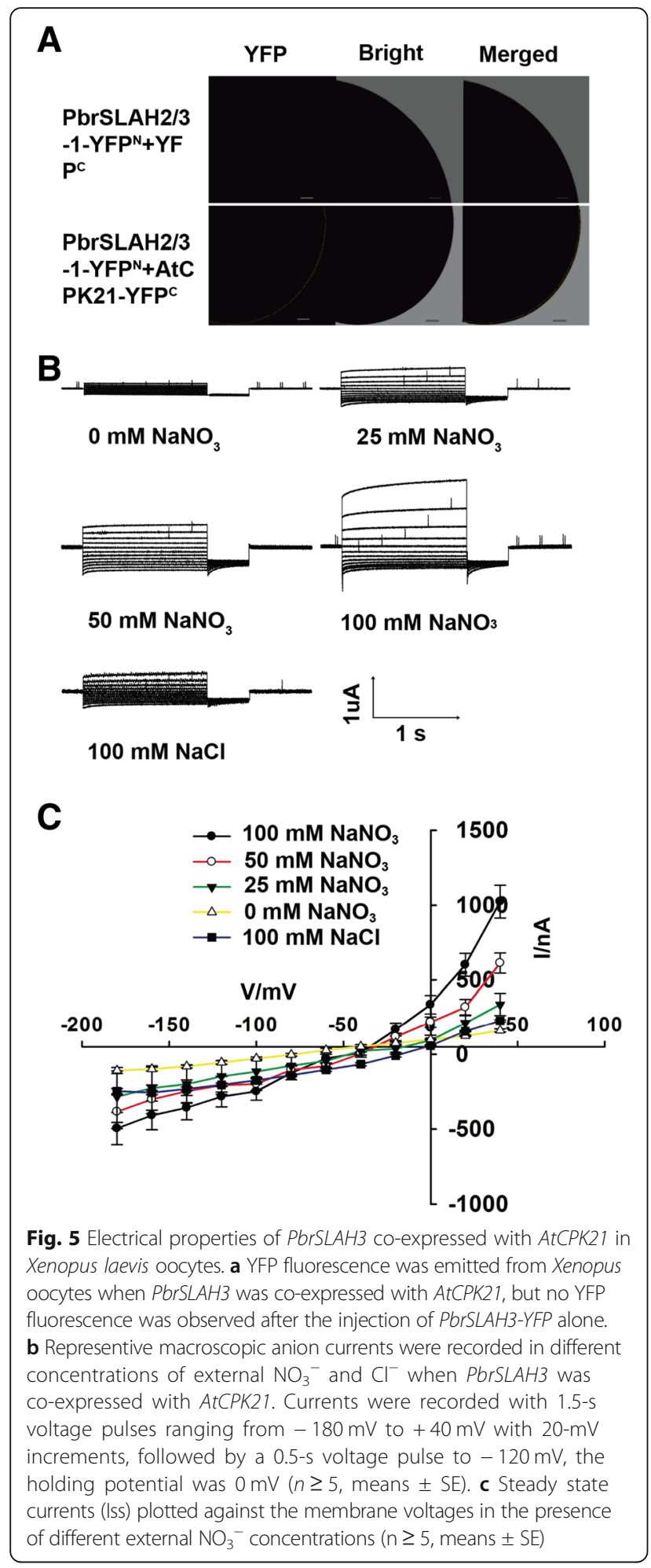

expressed in root, are targets for $\mathrm{NO}_{3}{ }^{-}$translocation in Arabidopsis root or from the root to shoot.

Based on previous reports about SLAC/SLAH genes expression patterns and functional characteristics $[12,28]$, we hypothesized that some members of

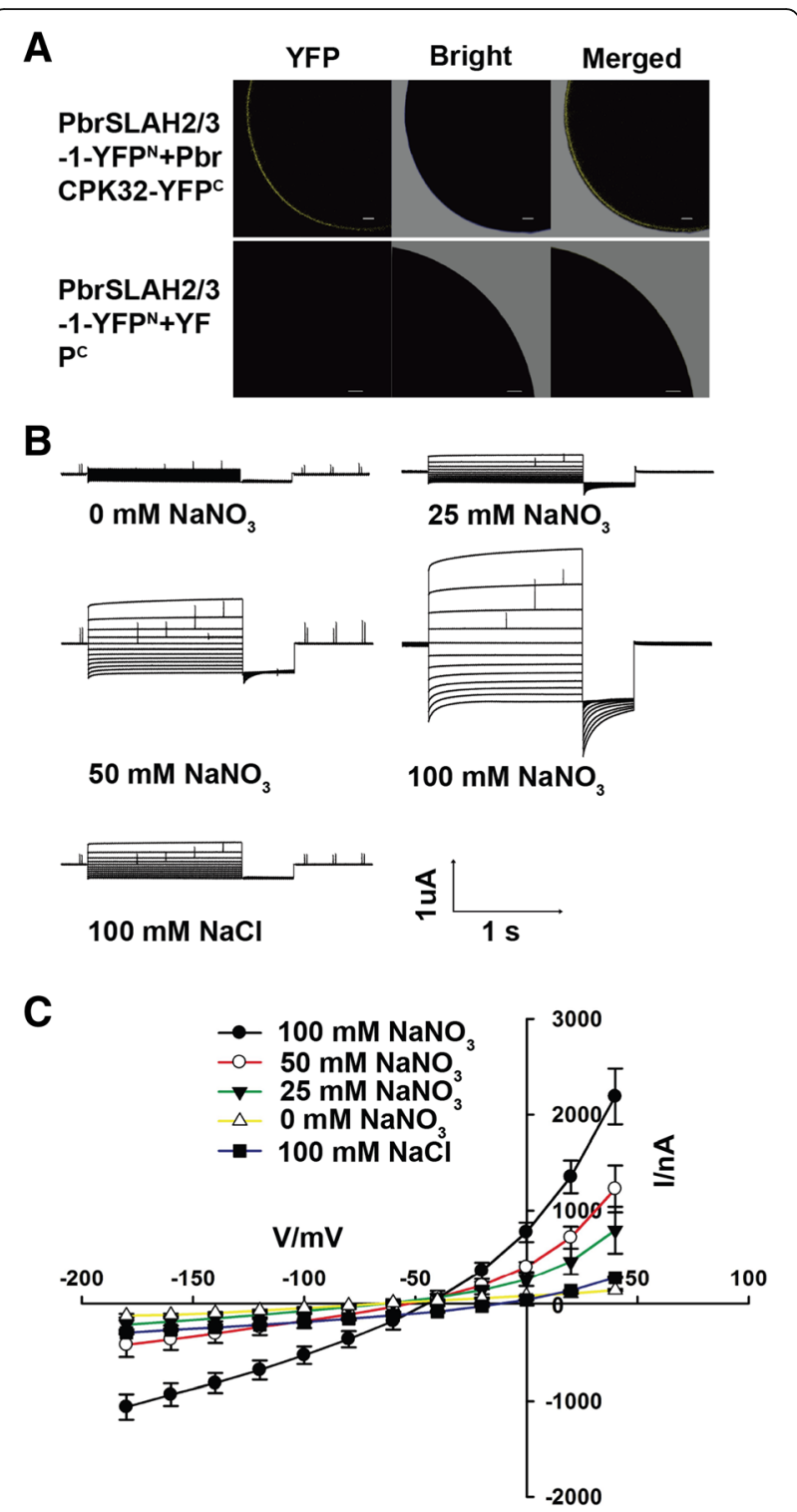

Fig. 6 Electrical properties of PbrSLAH3 co-expressed with PbrCPK32 in Xenopus oocytes. a YFP fluorescence was observed in Xenopus oocytes when PbrSLAH3 was co-expressed with PbrCPK32, while no YFP fluorescence was detected when PbrSLAH3-YFP cRNA alone was injected. b Representive macroscopic anion currents were recorded in different concentrations of external $\mathrm{NO}_{3}{ }^{-}$and $\mathrm{Cl}^{-}$when PbrSLAH3 was co-expressed with PbrCPK32. Currents were recorded with 1.5-s voltage pulses ranging from $-180 \mathrm{mV}$ to $+40 \mathrm{mV}$ with $20-\mathrm{mV}$ increments, followed by a 0.5 -s voltage pulse to $-120 \mathrm{mV}$, the holding potential was $0 \mathrm{mV}$. ( $n \geq 5$, means \pm SE). c Steady state currents (Iss) plotted against the membrane voltages in the presence of different external $\mathrm{NO}_{3}{ }^{-}$concentrations ( $n \geq 5$, means $\pm \mathrm{SE}$ )

the $S L A C / S L A H$ family in pear may be involved in nitrate transport in root or from the root to the shoot, and may play an important role in determining the nitrogen use efficiency of the pear. In this study, four putative SLAC/SLAH genes were identified in the Pyrus genome. Surprisingly, it was found 
that orthologs of AtSLAC/SLAH in pear did not phylogenetically cluster with AtSLAH1 and AtSLAH4. While, the expression patterns of PbrSLAC/SLAH are consistent with previous reports [25], PbrSLAC1 is strongly expressed in leaves, and PbrSLAH3 displays a higher expression level than other $S L A H$ genes in pear root. Therefore, we presumed that PbrSLAH3 may predominantly participate in $\mathrm{NO}_{3}{ }^{-}$transport in pear root. Here, the functional characteristics of PbrSLAH3 were mainly studied using complementation lines and a Xenopus oocyte heterogeneous expression system.

The predominant expression of PbrSLAH3 in root was further confirmed by RT-PCR and GUS staining assays, which demonstrated that it was also expressed in guard cells and pollen tubes, suggesting that PbrSLAH3 may have diverse functions similar to those of AtSLAH3. Furthermore, phenotype assays showed that PbrSLAH3 also rescues the ammonium toxicity phenomenon of the slah3-3 mutant under low $\mathrm{NO}_{3}{ }^{-} /$high $\mathrm{NH}_{4}{ }^{+}$conditions similar to that of the AtSLAH3. Taken together, we presumed that PbrSLAH3 together with AtSLAH3 and other orthologes from different species may be used as promising targets for crop enhancement, and they may serve as potential targets for transgenic breeding approaches to improve nitrogen use efficiency and reduce dependency on nitrogen fertilizers.

Post-translational modifications of transport proteins play key roles in the regulation of anion transport activities. For instance, AtSLAC1 and AtSLAH3 were activated by the $\mathrm{Ca}^{2+}$-independent protein kinase OST1 [29-31], as well as by AtCPKs [24, 29, 32]. SLAH2 was activated by CPK21 and CIPK23 kinases [22]. In this study, PbrSLAH3 was confirmed to interact with PbrCPK32 through yeast two-hybrid and BiFC assays. Furthermore, the electrophysiological experiments demonstrated that PbrSLAH3's activation requires phosphorylation. When PbrSLAH3 alone injection into Xenopus oocytes, no currents were elicited, but when PbrSLAH3 and $A t C P K 21$ were co-expressed in the Xenopus oocytes, typical anion currents were recorded in the presence of $\mathrm{NO}_{3}{ }^{-}$. However, not all isolated anion channels from different species dependent on the phosphorylation process. For example, PttSLAH3 isolated from poplar is activated independent of phosphorylation by protein kinases [10]. In addition, the silent SLAH1 subunit gates $S L A H 3$ open even in the absence of calcium-dependent kinases when co-expression the SLAH1 and SLAH3 in Xenopus oocytes [20]. In line with the function characteristic of the AtSLAH3, PbrSLAH3 was strongly dependent on the external concentration of $\mathrm{NO}_{3}{ }^{-}$and showed a high selectivity for nitrate over chloride. However, the biological functions of PbrSLAH3 and PbrCPK32 in pear are still unknown.

\section{Conclusions}

S-type anion channels play an important role in nitrate translocation, revealing another manner to expand the transport of nitrate anions. However, the function characteristics of PbrSLAC/SLAH in pear have not been clarified. In this study, four members of S-type anion channels were identified in pear using Arabidopsis $S L A C / S L A H$ sequences as queries. In combination with homologous similarity and expression analyses, PbrSLAH3 was selected as a candidate gene involved in $\mathrm{NO}_{3}{ }^{-}$transport in pear root. The physiological function of PbrSLAH3 was investigated by complementation lines, growth assays suggested that PbrSLAH3 participates in the alleviation of ammonium toxicity. PbrSLAH3 interaction with PbrCPK32 was firstly confirmed by yeast two-hybrid and BiFC assays, and PbrSLAH3 activity was controlled through phosphorylation by CPKs was further verified by co-expression it with AtCPK21 or PbrCPK32 in Xenopus oocytes. Furthermore, electrophysiological and physiology experiment results showed that PbrSLAH3 was more permeable to $\mathrm{NO}_{3}{ }^{-}$than $\mathrm{Cl}^{-}$. Taken together these results, we concluded that PbrSLAH3 crossing-talk with PbrCPK32 may participate in transporting $\mathrm{NO}_{3}{ }^{-}$in pear root.

\section{Methods}

\section{Plant materials and growth conditions}

The Columbia (Col-0) line of Arabidopsis was used as the wild-type (Col-0). The slah3-3 (SALK_106054) mutant seed was ordered from the Arabidopsis Biological Resource Center. Homozygous mutant plants were isolated by PCR with corresponding primers, as listed in Additional file 1: Table S1. For seed harvest, Arabidopsis plants were grown in a potting soil mixture (rich soil:vermiculite $=2: 1, \mathrm{v} / \mathrm{v}$ ) and kept in growth chambers with a light/dark regime of $14 / 10 \mathrm{~h}$, at $28 / 22{ }^{\circ} \mathrm{C}$ with $75 \%$ relative humidity and a light intensity of $800 \mu \mathrm{mol} \mathrm{m}{ }^{-2}$ $\mathrm{s}^{-1}$. For nitrate treatment experiments, surface-sterilized seeds were germinated and grown on 1/2 N-free Murashige and Skoog medium ( $\mathrm{pH}$ 5.8) containing different concentrations $(0-20 \mathrm{mM})$ of $\mathrm{KNO}_{3}$ with or without 20 $\mathrm{mM}$ ammonium chloride. Plates were kept at $4{ }^{\circ} \mathrm{C}$ for 3 $\mathrm{d}$ before seed germination. All plants were grown vertically under axenic conditions in growth chambers as described above. Plants were photographed with a Canon EOS 80D camera (Canon, Tokyo, Japan). Statistical analyses of the data were performed using the SPSS statistical package (SPSS Version 17.0 for Windows; SPSS Inc., Chicago, IL).

\section{Bioinformatics analysis of PbrSLAC/PbrSLAH}

The protein sequences of AtSLAC1 (AT1G12480), AtSLAH1 (AT1G62280), AtSLAH2 (AT4G27970), AtSLAH3 (AT5G24030) and AtSLAH4 (AT1G62262) were used as queries to perform a BLAST algorithm-based 
search of the pear genome database (http://peargenome. njau.edu.cn/). Based on our previous research, four PbrSLAC/SLAH protein sequences were identified [33]. They were aligned with orthologs in Arabidopsis, and a phylogenetic tree was generated by MEGA (version 6.0) software with the neighbor-joining method and a bootstrap test of 1000 replicates [34].

\section{RNA isolation and qRT-PCR assays}

Total RNA was extracted from different parts (root, stem, leaf, flower, fruit, pollen grain and pollen tube) of pear using an RNA extracting kit (RNAsimply Total RNA Kit, Tiangen, Beijing, China) according to the manufacturer's protocol. Then approximately $2 \mu \mathrm{g}$ total RNA was used for first-strand cDNA synthesis using TransScript ${ }^{\circ}$ One-Step gDNA Removal and cDNA Synthesis SuperMix (TRANSGEN, Beijing, China). Three biological and three technical replicates were processed for the qRT-PCR assays, which was performed in $20 \mu \mathrm{L}$ reaction mixture containing 80-100 ng cDNA, $200 \mathrm{nM}$ of each primer (Additional file 1: Table S1) and $10 \mu \mathrm{L}$ LightCycler 480 SYBRGREEN I Master Mix (Roche, Basel, Switzerland). All reactions were carried out in a CFX96 Real-Time System (Roche), following a three step standard protocol $\left(45\right.$ cycles of $10 \mathrm{~s}$ at $95^{\circ} \mathrm{C}, 30 \mathrm{~s}$ at $60^{\circ} \mathrm{C}$ and $30 \mathrm{~s}$ at $72{ }^{\circ} \mathrm{C}$ ), followed by a melt curve analysis. The expression levels of PbrSLAC/SLAH genes were calibrated by the $2^{-\triangle \Delta C t}$ method using $P b r U B Q$ and PbrTUB as reference genes [35]. RT-PCR was carried out on a Veriti ${ }^{\text {Tw }}$ 96-Well Thermal cycler (Bio-Rad, Richmond, CA, USA) using Taq DNA Polymerase (New England Biolabs, Beverly, MA, USA). PbrUBQ was used as the reference gene. PCR products were separated on a $2 \%(\mathrm{w} / \mathrm{v})$ agarose gel stained with ethidium bromide.

\section{Subcellular localization assays}

To test the subcellular localization of PbrSLAH3 and its regulatory protein, the full-length coding sequences of PbrSLAH3 and PbrCPK32 were amplified with the corresponding primers, respectively, which are listed in Additional file 1: Table S1. The purified PCR products were independently inserted into the pCAMBIA1302 vector under the control of the CaMV $35 \mathrm{~S}$ promoter and the vector is detailed in xie et al [36]. The recombinant plasmids 35S:PbrSLAH3-green fluorescence protein (GFP) and 35S:PbrCPK32-GFP, as well as the control plasmid 35S:GFP, were independently introduced into Nicotiana benthamiana leaves by Agrobacteriummediated transformation. Transformed leaves were stained with double-distilled water supplemented with $5 \mu \mathrm{M}$ FM4-64 (Invitrogen) for $15 \mathrm{~min}$, and the GFP signal was detected with a Zeiss LSM 780 Image Browser (Carl Zeiss Inc.) $3 \mathrm{~d}$ after transformation.

\section{BiFC assays}

The full-length cDNAs of PbrSLAH3 and PbrCPK32 were each inserted independently into the pYFP-N and pYFP-C vectors [37] and sequenced. Then, $N$. benthamiana leaves were transiently transformed using the Agrobacterium infection method with different combinations of these vectors. After $3 \mathrm{~d}$, the YFP expression signals emitted from $N$. benthamiana leaves were observed with a Zeiss LSM 780 Image Browser (Carl Zeiss Inc.).

\section{Yeast two-hybrid assays}

To screen for interactions between PbrSLAH3 and PbrCPKs, the coding sequence of PbrSLAH3 was cloned into the pDHB-C vector, and the full-length $P b r C P K$ cDNAs were independently cloned into the $\mathrm{pPR} 3-\mathrm{N}$ vector [38]. Next, PbrSLAH3-pDHB-C and PbrCPK-pPR3-N plasmids were co-transformed into the yeast strain NMY51 using the lithium acetate method. NubG co-transformed with $\mathrm{pDHB}-\mathrm{C}$ was used as a positive control, and NubG co-transformed with pPR3-N was used as a negative control. Then, co-transformed yeast cells were grown on selective medium lacking leucine and tryptophan (SD/-Trp-Leu) and were cultured at $28^{\circ} \mathrm{C}$. Colonies from $\mathrm{SD} /$-Trp-Leu plates were transferred to plates lacking leucine, tryptophan, histidine and adenine (SD/-Trp-Leu-His-Ade) and dyed with X-gal to test for possible interactions.

\section{Vector construction and Arabidopsis transformation}

The PbrSLAH3 promoter fragment $(\sim 2 \mathrm{~kb})$ was cloned into the pBasta-GUSGW vector [39], and PbrSLAH3 was cloned into the pCAMBIA1302 vector [36]. Then, PbrSLAH3::GUS and PbrSLAH3-pCAMBIA1302 recombination vectors were transformed into Col-0 Arabidopsis plants and slah3 mutant plants, respectively. The Arabidopsis transformation was performed using the floral dip method with Agrobacterium GV3101 [40]. The seeds of the transgenic plants were collected and then plated on half-strength MS medium containing $30 \mathrm{mg} / \mathrm{L}$ of hygromycin. Positive seedlings were verified by PCR with primers listed in Additional file 1: Table S1, and T3 homozygous transgenic lines were used for the later GUS assays and phenotypic analysis.

\section{GUS assays}

GUS staining was carried out according to the method described by Jefferson et al $[40,41]$. Two-week-old seedlings, flowers and siliques were vacuum-infiltrated for $30 \mathrm{~min}$ in the staining solution (GUS staining kit, Pulangsai, Beijing, China) followed by incubation at $37^{\circ} \mathrm{C}$ for $3 \mathrm{~h}$. After gradient decolorization by alcohol, the samples were photographed under a microscope with a Canon EOS 80D camera (Canon, Tokyo, Japan). 
Double-electrode voltage-clamp studies (oocyte recordings) PbrSLAH3 cDNA fused with pYFP-C and PbrCPK32 cDNA fused with pYFP-N were independently cloned into the pT7TS vector using homologous recombination technology [42]. The capped and polyadenylated cRNA of these two fusion genes were synthesized in vitro using a MESSAGE kit (Ambion, Austin, Texas, USA). Then, $50 \mathrm{~nL}$ cRNA mixture of the target genes (PbrSLAH3-YFP-C and PbrCPK32-YFP-N) or $50 \mathrm{~nL}$ of deionized water (control) was injected into matured oocytes, which were then cultured in 'ND96' solution $(96 \mathrm{mM} \mathrm{NaCl}, 2$ $\mathrm{mM} \mathrm{KCl}, 1.8 \mathrm{mM} \mathrm{CaCl}, 1 \mathrm{mM} \mathrm{MgCl}_{2}$ and $5 \mathrm{mM}$ HEPES, adjusted to pH 7.4 with $\mathrm{NaOH}$ ) until electrophysiological recordings were taken. Then, 3-5 d after cRNA injections, whole-oocyte currents were recorded using the two-electrode voltage-clamp technique. The voltage-clamp amplifier was an Axoclamp 900A (Axon Instruments, Foster City, CA, USA). Electrodes were filled with $3 \mathrm{M} \mathrm{KCl}$, and the standard solution contained $5 \mathrm{mM}$ Tris/MES (pH 7.5), $1 \mathrm{mM} \mathrm{Ca(gluconate)})_{2}, 1 \mathrm{mM}$ $\mathrm{Mg}$ (gluconate) ${ }_{2}, 100 \mathrm{mM} \mathrm{NaNO}$ and $1 \mathrm{mM} \mathrm{LaCl}_{3}$. To balance the ionic strength, nitrate or chloride variations were compensated with sorbitol. Osmolality was adjusted to $220 \mathrm{mos}-\mathrm{mol} / \mathrm{kg}$ with D-sorbitol. The voltage-clamp protocols are described in the figure legends, and data acquisition and data analyses were performed using pClampfit 10.3 (Molecular Devices) and Sigmaplot 12.5 software (Jandel Scientific, Erkrath, Germany), respectively. Absorption or transport.

\section{Determination of the $\mathrm{NO}_{3}{ }^{-}$and $\mathrm{Cl}^{-}$contents}

The 35S:PbrSLAH3 and slah3-3 mutant Arabidopsis seeds were plated on solid 1/2 MS medium. Two-week-old seedlings were pre-cultured in the 72 holes rectangular box containing $500 \mathrm{~mL}$ of liquid 1/2 MS medium for $12 \mathrm{~d}$. In the process of pre-culture, the nutrient solution was replaced with fresh solution every $4 \mathrm{~d}$. After the pre-culture, seedlings were treated with the $1 / 2 \mathrm{NO}_{3}{ }^{-}$-free and $\mathrm{Cl}^{-}$-free MS for 3 days, and then were transferred into modified liquid $1 / 2 \mathrm{MS}$ containing $5 \mathrm{mM} \mathrm{NO}_{3}{ }^{-}$and $5 \mathrm{mM} \mathrm{Cl}^{-} .180$ seedlings were used per treatment, with three replications. Concentrations of $\mathrm{NO}_{3}{ }^{-}$and $\mathrm{Cl}^{-}$were determined in the liquid $1 / 2 \mathrm{MS}$ solution at $0 \mathrm{~h}, 6 \mathrm{~h}, 12 \mathrm{~h}, 24 \mathrm{~h}, 48 \mathrm{~h}$ and $96 \mathrm{~h}$ after the transferring, respectively, which are based on the method described by Cataldo et al [43] and Gilliam et al [44]. In addition, the volume of nutrient solution needs to be replenished to $500 \mathrm{~mL}$ with double distilled water before each sampling. Statistical analyses of the data were performed using the SPSS statistical package (SPSS Version 17.0 for Windows; SPSS Inc., Chicago, IL). Three biological replicates and three technical replicates were processed, and all plants were grown in chambers with a light/dark regime of $14 / 10 \mathrm{~h}$, at $28 / 22^{\circ} \mathrm{C}$ with $75 \%$ relative humidity and a light intensity of $800 \mu \mathrm{mol} \mathrm{m}^{-2} \mathrm{~s}^{-1}$.

\section{Additional file}

\begin{abstract}
Additional file 1: Figure S1. Changes in PbrSLAH3 expression in response to re-supplying nitrate in pear root after nitrate starvation. Figure S2. PbrSLAH3 rescues the ammonium toxicity of slah3-3 mutant plants under high-ammonium/low-nitrate conditions. Figure S3. PbrSLAH3 interaction with AtCPK21 was confirmed by bimolecular fluorescence complementation assays. Figure S4. Phylogenetic tree of calcium-dependent proteins in pear and in Arabidopsis. Figure S5. The interaction between PbrSLAH3 and PbrCPKs was assessed by the yeast two-hybrid assays. Figure S6. The subcellular localization of PbrCPK32 in plasma membrane and the expression profiles of the PbrCPK32 genes in different parts of the pear were analyzed by qRT-PCR. Figure S7. No fluorescence was detected when PbrSLAH3 or PbrCPK32 was expressed alone in $\mathbf{N}$. benthamiana leaves (negative control). Figure S8. Representive macroscopic anion currents were recorded in $50 \mathrm{mM} \mathrm{NaNO}_{3}$ solution after independently injects of $\mathrm{H}_{2} \mathrm{O}$ and $\mathrm{PbrSLAH}_{3}$ alone. Figure S9. Comparative analysis of $\mathrm{NO}_{3}$ and $\mathrm{Cl}$ transport between 35S:PbrSLAH3 and slah3-3 mutant plants. Table S1. Primers used in this study. Table S2. CPK family genes identified in pear. (PDF $1355 \mathrm{~kb}$ )
\end{abstract}

\section{Abbreviations}

ABA: Abscisic acid; Ade: Adenine; BiFC: Bimolecular fluorescence complementation; CPK: Calcium-dependent protein kinase; GFP: Green Fluorescent Protein; GUS: $\beta$-Glucuronidase; His: Leucine; Leu: Leucine; OST1: Stomatal opening factor 1; qRT-PCR: Quantitative real-time polymerase chain reaction; RT-PCR: Real-time polymerase chain reaction; Stype anion channels: S-type anion channels; Trp: Tryptophan; YFP: Yellow Fluorescent Protein

\section{Acknowledgements}

Not applicable.

\section{Funding}

The National Key R\&D Program of China (2018YFD0201400) and the National Key Technology R\&D Program of the Ministry of Science and Technology of China (2014BAD16B03-4) were supported for designing the study and carrying out the experiments, the Fundamental Research Funds for the Central Universities (KJQN201926 and KYTZ201602) was supported for analysis and interpretation of data, and the National Natural Science Foundation of China (31801842) was supported for polish the manuscript.

Availability of data and materials

The data and material that support the findings of this study are available from the corresponding author on request.

\section{Authors' contributions}

CGD designed the research, performed the experiments, and analyzed the results. WL and WJY drafted the manuscript. CQ, CP, TC and WX participated in carrying out the experiments. WJY, WL, QKJ, YH, ZSL, and WP participated in revising the final manuscript. WJY managed the experiments. All authors have read and approved the final manuscript.

Ethics approval and consent to participate Not applicable.

Consent for publication Not applicable.

Competing interests

The authors declare that they have no competing interests.

\section{Publisher's Note}

Springer Nature remains neutral with regard to jurisdictional claims in published maps and institutional affiliations. 


\section{Received: 17 August 2018 Accepted: 30 April 2019}

\section{Published online: 08 May 2019}

\section{References}

1. Zimmermann S, Ehrhardt T, Plesch G, Muller-Rober B. Ion channels in plant signaling. Cell Mol Life Sci. 1999;55(2):183-203.

2. Czempinski K, Gaedeke N, Zimmermann S, Muller-Rober B. Molecular mechanisms and regulation of plant ion channels. J Exp Bot. 1999;50:955-66.

3. Blatt MR, Thiel G. Hormonal-control of Ion-Channel gating. Annu Rev Plant Phys. 1993:44:543-67.

4. Tyerman SD. Anion channels in plants. Annu Rev Plant Phys. 1992:43:351-73.

5. Neher E, Sakmann B. The patch clamp technique. Sci Am. 1992;266(3):44-51.

6. Keller BU, Hedrich R, Raschke K. Voltage-dependent anion channels in the plasma-membrane of guard-cells. Nature. 1989;341(6241):450-3.

7. Schroeder Jl, Hagiwara S. Cytosolic calcium regulates ion channels in the plasma-membrane of Vicia-Faba guard-cells. Nature. 1989;338(6214):427-30.

8. Vahisalu T, Kollist H, Wang YF, Nishimura N, Chan WY, Valerio G, Lamminmaki A, Brosche M, Moldau H, Desikan R, et al. SLAC1 is required for plant guard cell Stype anion channel function in stomatal signalling. Nature. 2008; 452(7186):487-91.

9. Negi J, Matsuda O, Nagasawa T, Oba Y, Takahashi H, Kawai-Yamada M, Uchimiya H, Hashimoto M, Iba K. CO2 regulator SLAC1 and its homologues are essential for anion homeostasis in plant cells. Nature. 2008;452(7186): 483-U413

10. Jaborsky M, Maierhofer T, Olbrich A, Escalante-Perez M, Muller HM, Simon J, Krol E, Cuin TA, Fromm J, Ache P, et al. SLAH3-type anion channel expressed in poplar secretory epithelia operates in calcium kinase CPK-autonomous manner. New Phytol. 2016;210(3):922-33.

11. Sun SJ, Qi GN, Gao QF, Wang HQ, Yao FY, Hussain J, Wang YF. Protein kinase OsSAPK8 functions as an essential activator of S-type anion channel OsSLAC1, which is nitrate-selective in rice. Planta. 2016;243(2):489-500.

12. Geiger D, Scherzer S, Mumm P, Stange A, Marten I, Bauer H, Ache P, Matschi S, Liese A, Al-Rasheid KA, et al. Activity of guard cell anion channel SLAC1 is controlled by drought-stress signaling kinase-phosphatase pair. Proc Natl Acad Sci U S A. 2009;106(50):21425-30.

13. Geiger D, Maierhofer T, AL-Rasheid KAS, Scherzer S, Mumm P, Liese A, Ache P, Wellmann C, Marten I, Grill E, et al. Stomatal closure by fast abscisic acid signaling is mediated by the guard cell anion channel SLAH3 and the receptor RCAR1. Sci Signal. 2011:4(173):ra32.

14. Wang YY, Hsu PK, Tsay YF. Uptake, allocation and signaling of nitrate. Trends Plant Sci. 2012;17(8):458-67.

15. Dreyer I, Gomez-Porras JL, Riano-Pachon DM, Hedrich R, Geiger D. Molecular evolution of slow and quick anion channels (SLACs and QUACS/ALMTS). Front Plant Sci. 2012:3:263.

16. Desikan R, Griffiths R, Hancock J, Neill S. A new role for an old enzyme: nitrate reductase-mediated nitric oxide generation is required for abscisic acid-induced stomatal closure in Arabidopsis thaliana. Proc Natl Acad Sci U S A. 2002:99(25):16314-8

17. Yoshida R, Umezawa T, Mizoguchi T, Takahashi S, Takahashi F, Shinozaki K. The regulatory domain of SRK2E/OST1/SnRK2.6 interacts with ABI1 and integrates abscisic acid (ABA) and osmotic stress signals controlling stomatal closure in Arabidopsis. J Biol Chem. 2006:281(8):5310-8.

18. Chen $\mathrm{YH}, \mathrm{Hu} L$, Punta $M$, Bruni $R$, Hillerich B, Kloss B, Rost B, Love J, Siegelbaum SA, Hendrickson WA. Homologue structure of the SLAC anion channel for closing stomata in leaves. Nature. 2010;467(7319): 1074-80.

19. Demir F, Horntrich C, Blachutzik JO, Scherzer S, Reinders Y, Kierszniowska S Schulze WX, Harms GS, Hedrich R, Geiger D, et al. Arabidopsis nanodomaindelimited ABA signaling pathway regulates the anion channel SLAH3. Proc Natl Acad Sci U S A. 2013;110(20):8296-301.

20. Cubero-Font P, Maierhofer T, Jaslan J, Rosales MA, Espartero J, Diaz-Rueda P, Muller HM, Hurter AL, AL-Rasheid KAS, Marten I, et al. Silent S-type anion channel subunit SLAH1 gates SLAH3 open for chloride root-to-shoot translocation. Curr Biol. 2016;26(16):2213-20.

21. Qiu JE, Henderson SW, Tester M, Roy SJ, Gilliham M. SLAH1, a homologue of the slow type anion channel SLAC1, modulates shoot Cl- accumulation and salt tolerance in Arabidopsis thaliana. J Exp Bot. 2016;67(15):4495-505.

22. Maierhofer T, Lind C, Huttl S, Scherzer S, Papenfuss M, Simon J, Al-Rasheid $\mathrm{KA}$, Ache $\mathrm{P}$, Rennenberg $\mathrm{H}$, Hedrich $\mathrm{R}$, et al. A single-pore residuerenders the Arabidopsis root anion channel SLAH2 highly nitrate selective. Plant Cell. 2014;26(6):2554-67.
23. Winter D, Vinegar B, Nahal H, Ammar R, Wilson GV, Provart NJ. An "electronic fluorescent pictograph" browser for exploring and analyzing large-scale biological data sets. PLoS One. 2007;2(8):e718.

24. Gutermuth T, Lassig R, Portes MT, Maierhofer T, Romeis T, Borst JW, Hedrich R, Feijo JA, Konrad KR. Pollen tube growth regulation by free anions depends on the interaction between the anion channel SLAH3 and calcium-dependent protein kinases CPK2 and CPK20. Plant Cell. 2013;25(11):4525-43.

25. Zheng XJ, He K, Kleist T, Chen F, Luan S. Anion channel SLAH3 functions in nitrate-dependent alleviation of ammonium toxicity in Arabidopsis. Plant Cell Environ. 2015;38(3):474-86.

26. Stitt M, Muller C, Matt P, Gibon Y, Carillo P, Morcuende R, Scheible WR, Krapp A. Steps towards an integrated view of nitrogen metabolism. J Exp Bot. 2002;53(370):959-70.

27. Wolf-Rüdiger Scheible MS. Genome-wide reprogramming of primary and secondary metabolism, protein synthesis, cellular growth processes, and the regulatory infrastructure of Arabidopsis in response to nitrogen. Plant Physiol. 2004;136(1):2483-99.

28. Park H, Oh SJ, Han KS, Woo DH, Park H, Mannaioni G, Traynelis SF, Lee CJ. Bestrophin-1 encodes for the $\mathrm{Ca}^{2+}$-activated anion channel in hippocampal astrocytes. J Neurosci. 2009;29(41):13063-73.

29. Brandt B, Brodsky DE, Xue S, Negi J, Iba K, Kangasjarvi J, Ghassemian M, Stephan AB, Hu H, Schroeder JI. Reconstitution of abscisic acid activation of SLAC1 anion channel by CPK6 and OST1 kinases and branched ABI1 PP2C phosphatase action. Proc Natl Acad Sci U S A. 2012;109(26):10593-8.

30. Vahisalu T, Puzorjova I, Brosche M, Valk E, Lepiku M, Moldau H, Pechter P, Wang YS, Lindgren O, Salojarvi J, et al. Ozone-triggered rapid stomatal response involves the production of reactive oxygen species, and is controlled by SLAC1 and OST1. Plant J. 2010;62(3):442-53.

31. Imes D, Mumm P, Bohm J, Al-Rasheid KAS, Marten I, Geiger D, Hedrich R. Open stomata 1 (OST1) kinase controls R-type anion channel QUAC1 in Arabidopsis guard cells. Plant J. 2013;74(3):372-82.

32. Geiger D, Scherzer S, Mumm P, Marten I, Ache P, Matschi S, Liese A, Wellmann C, Al-Rasheid KA, Grill E, et al. Guard cell anion channel $\mathrm{SLAC1}$ is regulated by CDPK protein kinases with distinct $\mathrm{Ca}^{2+}$ affinities. Proc Natl Acad Sci U S A. 2010;107(17):8023-8.

33. Chen $\mathrm{G}$. The slow anion channel (SLAC/SLAH) gene family in pear (Pyrus bretschneideri) and comparison with other members of the Rosaceae. Genomics. 2018. https://doi.org/10.1016/j.ygeno.2018.07.004.

34. Tamura K, Dudley J, Nei M, Kumar S. MEGA4: molecular evolutionary genetics analysis (MEGA) software version 4.0. Mol Biol Evol. 2007;24(8):1596-9.

35. Livak KJ, Schmittgen TD. Analysis of relative gene expression data using real-time quantitative PCR and the 2(T)(-Delta Delta C) method. Methods. 2001;25(4):402-8.

36. Xie QG, Wang P, Liu X, Yuan L, Wang LB, Zhang CG, Li Y, Xing HY, Zhi LY, Yue $Z \mathrm{~L}$, et al. LNK1 and LNK2 are transcriptional coactivators in the Arabidopsis circadian oscillator. Plant Cell. 2014;26(7):2843-57.

37. Sun L, Song L, Zhang Y, Zheng Z, Liu D. Arabidopsis PHL2 and PHR1 act redundantly as the key components of the central regulatory system controlling transcriptional responses to phosphate starvation. Plant Physiol. 2016;170(1):499.

38. Dong JS, Pineros MA, Li XX, Yang HB, Liu Y, Murphy AS, Kochian LV, Liu D. An Arabidopsis ABC transporter mediates phosphate deficiency-induced remodeling of root architecture by modulating iron homeostasis in roots. Mol Plant. 2017;10(2):244-59.

39. Xie XB, Zhao J, Hao YJ, Fang CB, Wang Y. The ectopic expression of apple MYB1 and bHLH3 differentially activates anthocyanin biosynthesis in tobacco. Plant Cell Tiss Org. 2017;131(1):183-94.

40. Clough SJ, Bent AF. Floral dip: a simplified method for Agrobacteriummediated transformation of Arabidopsis thaliana. Plant J. 1998;16(6):735-43.

41. Jefferson RA, Kavanagh TA, Bevan MW. GUS fusions $\beta$-glucuronidase as a sensitive and versatile gene fusion marker in higher-plants. EMBO J. 1987; 6(13):3901-7.

42. Mei W, Song W, Pan Y, Gong W, Zhu Y. High throughput cloning of Arabidopsis transcription factors using gateway cloning technology. Mol Plant Breed. 2004;2(3):358-64.

43. Cataldo D, Maroon M, Schrader L, Youngs V. Rapid colorimetric determination of nitrate in plant tissue by nitration of salicylic acid. Commun Soil Sci Plan. 1975;6(1):71-80.

44. Gilliam JW. Rapid measurement of chlorine in plant materials. Soil Sci Soc Am J. 1971;35(3):512-3. 